American Journal of Biochemistry and Biotechnology 4 (4): 345-353, 2008

ISSN 1553-3468

(C) 2008 Science Publications

\title{
A Review on Supercritical Fluid Extraction as New Analytical Method
}

\author{
${ }^{1}$ Abbas, K.A., ${ }^{1}$ A. Mohamed, ${ }^{1}$ A.S. Abdulamir and ${ }^{2}$ H.A. Abas \\ ${ }^{1}$ Department of Food Technology, Faculty Food Science and Technology, \\ University Putra Malaysia (UPM), Malaysia \\ ${ }^{2}$ Department of English, Faculty of Arts, University of Imam Al-sadiq, Iraq
}

\begin{abstract}
This review study summarized the Supercritical Fluid Extraction (SFE) as new analytical method. The advantage and disadvantage of $\mathrm{CO}_{2}\left(\mathrm{SC}-\mathrm{CO}_{2}\right)$ as well as special applications of SFE in food processing such as removal of fat from food, enrichment of vitamin $\mathrm{E}$ from natural sources, removal of alcohol from wine and beer, encapsulation of liquids for engineering solid products and extraction and characterization of functional compounds were also highlighted. The study also covered the application of SFE in food safety such as rapid analysis for fat content, rapid analysis for pesticides in foods as well as the recent applications of SFE in food such as supercritical particle formation and nan particle formation and ssupercritical drying. The outcome finding confirmed that SFE was found to be advance, fast, reliable, clean and cheap methods for routine food analysis.
\end{abstract}

Key words: Supercritical fluid, applications, features

\section{INTRODUCTION}

SFE is used on a large scale for the extraction of some food grade and essential oils and pharmaceutical products from plants. It is relatively rapid because of the low viscosities and high diffusivities associated with supercritical fluids. The extraction can be selective to some extent by controlling the density of the medium and the extracted material is easily recovered by simply depressurizing, allowing the supercritical fluid to return to gas phase and evaporate leaving no or little solvent residues $^{[1]}$. However, carbon dioxide is the most common supercritical solvent.

Since the end of the 1970s, supercritical fluids has been used to isolate natural products, but for a long time the applications concentrated on only few products. Now the development of processes and equipment is beginning to pay off and industries are getting more and more interested in supercritical techniques $^{[2]}$.

In $\mathrm{SF}$ the physicochemical properties of a given fluid, such as density, diffusivity, dielectric constant and viscosity can be easily controlled by changing the pressure or the temperature without ever crossing phase boundaries ${ }^{[1]}$. The phase diagram of a single substance can be seen in Fig. 1.

The dissolving power of a supercritical fluid depends on its density, which unlike that of liquid solvents is highly adjustable by changing pressure or temperature. Especially in the vicinity of the critical point a small isothermal raise in pressure increases the fluid density remarkably while the effect diminishes with increasing distance from the critical values. Thus, the solvent strength of a fluid can be controlled more easily than that of a liquid solvent ${ }^{[1]}$. Recently, the supercritical fluid has approved higher diffusion coefficient and lower viscosity and surface tension than a liquid solvent, which leads to a more favorable mass transfer ${ }^{[3]}$.

The demand for new analytical techniques in food and environmental sciences is strongly related to the higher demand of information on processing, quality control, adulteration, contamination, environmental and food regulations and the need for faster, more powerful, cleaner and cheaper analytical procedures are required by chemists, regulatory agencies and quality control laboratories to meet these demands can be achieved by SFE. Thus, this review paper has been justified.

Properties and fundamentals of supercritical fluids Solvent strength: The density of a supercritical fluid is extremely sensitive to minor changes in temperature and pressure near the critical point. The densities of the fluids are closer to that of organic liquids but the solubility of solids can be 3-10 orders of magnitude higher. The solvent strength of a fluid can be expressed by the solubility parameter, which is the square root of the cohesive energy density and is defined rigorously

Corresponding Author: Abbas, K.A., Department of Food Technology, Faculty Food Science and Technology, University Putra Malaysia (UPM), Malaysia 


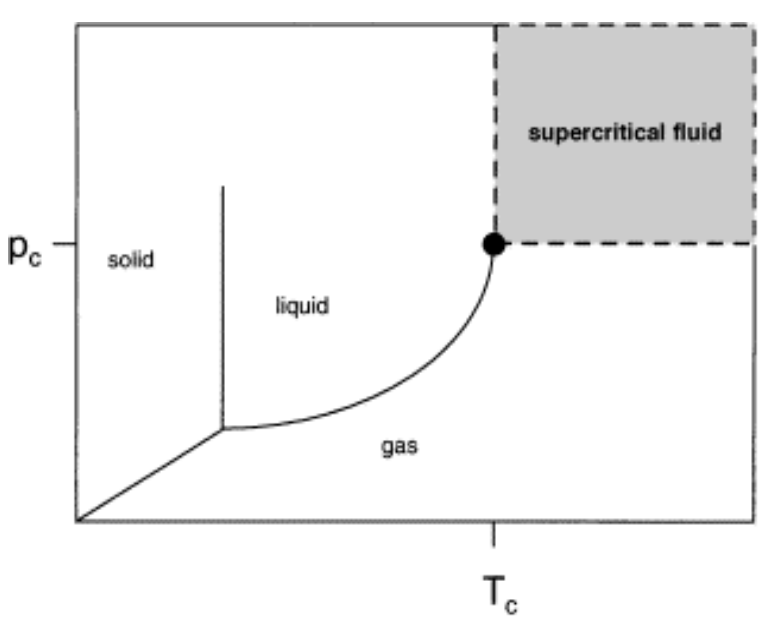

Fig. 1: Phase diagram for a single substance. $P_{c}$, critical pressure; $T_{c}$, critical temperature ${ }^{[1]}$

from first principles. A plot of the solubility parameter for carbon dioxide versus pressure would resemble that a plot of density versus pressure ${ }^{[3]}$. This confirms that the solvation strength of a supercritical fluid is directly related to the fluid density. Thus the solubility of a solid can be manipulated by making slight changes in temperatures and pressures. Another attractive feature of supercritical fluids is that the properties lie between that of gases and liquids. A supercritical fluid has densities similar to that of liquids, while the viscosities and diffusivities are closer to that of gases. Thus, a supercritical fluid can diffuse faster in a solid matrix than a liquid, yet possess a solvent strength to extract the solute from the solid matrix ${ }^{[4,2]}$.

Phase behavior: An understanding of the phase behavior is important since the phase behavior observed in supercritical fluids considerably differ from the behavior observed in liquids. One such behavior is the retrograde region. For an isobaric system, an increase in the temperature of a solution increases the solubility of the solute over certain ranges of pressure (consistent with the typical liquid systems) but decreases the solute solubility in other pressure ranges. This anomalous behavior wherein the solubility of the solute decreases with a temperature increase is called the retrograde behavior. Thus, the following generalizations may be made regarding the solute solubilities in supercritical fluids. Solute solubilities approach and may exceed that of liquid solvents. Solubilities generally increase with increase in pressure. An increase in the temperature of the supercritical fluid may increase, decrease or have no effect on the solubility of the solute depending upon the pressure $^{[1]}$.
$\mathrm{CO}_{2}$ is not a very good solvent for high molecular weight and polar compounds. To increase the solubility of such compounds in supercritical carbon dioxide, small amounts (ranging from 0 to $20 \mathrm{~mol} \%$ ) of polar or non-polar cosolvents called modifiers may be added. The co solvent interacts strongly with the solute and significantly increases the solubility ${ }^{[1,2]}$.

Co-solvents or modifiers: A more common practice in SFE is to change the polarity of the supercritical fluid and increase their solvating power towards the analyte of interest by employing polar modifiers (co-solvents). For example, the addition of relatively small percentages $(1-10 \%)$ of methanol to carbon dioxide expands its extraction range to include more polar analytes. The modifiers can also reduce the analytematrix interactions improving their quantitative extraction $^{[1]}$.

There are two main procedures to study with cosolvents or modifiers in SFE are; the first one, and the most common, accounts for a mixing of the modifier with the $\mathrm{CO}_{2}$ flow while the second mixes the modifier with raw material in the extraction cell. This procedure is always associated to a static extraction step in which the modifier, in intimate contact with the sample matrix, is able to substitute the analyte molecules bound in active centers of the matrix and release them into the supercritical fluid phase ${ }^{[5]}$.

Extraction with supercritical fluids: Supercritical extraction has been applied to a large number of solid matrices. The desired product can be either the extract or the extracted solid itself. The advantage of using supercritical fluids in extraction is the ease of separation of the extracted solute from the supercritical fluid solvent by simple expansion. In addition, supercritical fluids have liquid like densities but superior mass transfer characteristics compared to liquid solvents due to their high diffusion and very low surface tension that enables easy penetration into the porous structure of the solid matrix to release the solute $^{[2,6]}$.

Extraction of soluble species (solutes) from solid matrices takes place through four different mechanisms. If there are no interactions between the solute and the solid phase, the process is simple dissolution of the solute in a suitable solvent that does not dissolve the solid matrix ${ }^{[2]}$. If there are interactions between the solid and the solute, then the extraction process is termed as desorption and the adsorption isotherm of the solute on the solid in presence of the solvent determines the equilibrium. Most solids extraction processes, such as activated carbon regeneration, fall in this category. A 
third mechanism is swelling of the solid phase by the solvent accompanied by extraction of the entrapped solute through the first two mechanisms, such as extraction of pigments or residual solvents from polymeric matrices. The fourth mechanism is reactive extraction where the insoluble solute reacts with the solvent and the reaction products are soluble hence extractable, such as extraction of lignin from cellulose. Extraction is always followed by another separation process where the extracted solute is separated from the solvent $^{[4]}$.

Another important aspect in supercritical extraction relates to solvent/solute interactions. Normally the interactions between the solid and the solute determine the ease of extraction, i.e., the strength of the adsorption isotherm is determined by interactions between the adsorbent and the adsorbate. However, when supercritical fluids are used, interactions between the solvent and the solute affect the adsorption characteristics due to large negative partial molar volumes and partial molar enthalpies in supercritical fluids $^{[4,6]}$.

The thermodynamic parameters that govern the extraction are found to be temperature, pressure, the adsorption equilibrium constant and the solubility of the organic in supercritical fluid. Similar to the retrograde behavior of solubility in supercritical fluids, the adsorption equilibrium constants can either decrease or increase for an increase in temperature at isobaric conditions. This is primarily due to the large negative partial molar properties of the supercritical fluids. In addition to the above factors, the rate parameters like the external mass transfer resistances, the axial dispersion in the fluid phase, and the effective diffusion of the organics in the pores also play a crucial role in the desorption process. A thorough understanding of these governing parameters is important in the modeling of supercritical fluid extraction process and in the design, development and future scale-up of the process $^{[4]}$.

SFE process: A simplified process-scale SFE system is shown in Fig. 2 and a typical batch extraction proceeds as follows. Raw material is charged in the extraction tank which is equipped with temperature controllers and pressure valves at both ends to keep desired extraction conditions. The extraction tank is pressurized with the fluid by means of pumps, which are also needed for the circulation of the fluid in the system. From the tank the fluid and the solubilized components are transferred to the separator where the solvation power of the fluid is decreased by increasing the temperature, or more likely, decreasing the pressure of

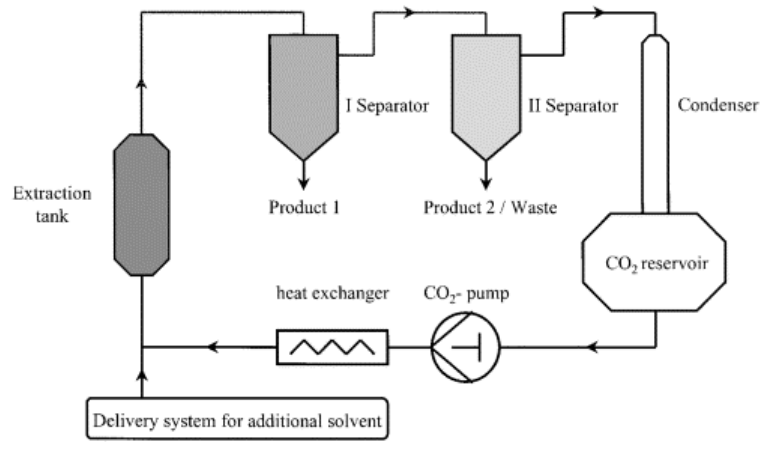

Fig. 2: A simplified drawing of a process-scale supercritical fluid extractor ${ }^{[1]}$.

the system. The product is then collected via a valve located in the lower part of the separator(s) ${ }^{[1,2]}$.

Advantages and drawbacks of supercritical $\mathrm{CO}_{2}$ $\left(\mathbf{S C}-\mathbf{C O}_{2}\right)$ : There are a large number of compounds that can be used as a fluid in supercritical techniques, but by far the most widely used is carbon dioxide. From the viewpoint of pharmaceutical, nutraceutical and food applications it is a good solvent, because it is non-toxic, non-flammable, inexpensive, easy to remove from the product and its critical temperature and pressure are relatively low $\left(\mathrm{T}_{\mathrm{c}}=31.1^{\circ} \mathrm{C}, \mathrm{p}_{\mathrm{c}}=72 \mathrm{bar}\right)$ make it important for food and natural products sample preparation, is the ability of SFE using $\mathrm{CO}_{2}$ to be operated at low temperatures using a non-oxidant medium, which allows the extraction of thermally labile or easily oxidized compounds. It is environmental friendly and generally recognized as safe by FDA and EFSA. These properties make it suitable for extracting, for example, thermally labile and non-polar bioactive compounds but, because of its non-polar nature, it cannot be used for dissolving polar molecules. The solubility of polar compounds and the selectivity of the process can be increased by adding small quantities of other solvents, such as ethanol, in the fluid that named as co-solvent or modifier. On one hand, it decreases the processing times, increases yields and makes it possible to use milder processing conditions, but on the other, it complicates system thermodynamics and increases capital costs ${ }^{[3]}$.

The use of high purity SFE-grade $\mathrm{CO}_{2}$ is not required but impurity and moisture in industrial grade $\mathrm{CO}_{2}$ can accumulate and may interfere with further analytical operations (gas or liquid chromatography). Thus, an on-line fluid cleanup system may be used to remove trace contaminants. An important drawback of $\mathrm{SC}-\mathrm{CO}_{2}$ and most of the other supercritical fluids is that predominantly, a non-polar extraction fluid, such as 
$\mathrm{CO}_{2}$, is used. Therefore, a logical trend to widen the application range of this technique is the study of new methods to decrease analyte polarity to make them more soluble in non-polar supercritical fluids. In this sense, chemical in situ derivatization has been applied to improve the selectivity of the extraction towards a specific group of compounds ${ }^{[3,5]}$. So the solvent power of $\mathrm{SC}-\mathrm{CO}_{2}$ can be summarized by a few rules:

- It dissolves non-polar or slightly polar compounds.

- The solvent power for low molecular weight compounds is high and decreases with increasing molecular weight.

- $\mathrm{SC}-\mathrm{CO}_{2}$ has high affinity with oxygenated organic compounds of medium molecular weight.

- Free fatty acids and their glycerides exhibit low solubilities.

- Pigments are even less soluble.

- Water has a low solubility $(<0.5 \% \quad \mathrm{w} / \mathrm{w})$ at temperatures below $100^{\circ} \mathrm{C}$.

- Proteins, polysaccharides, sugars and mineral salts are insoluble and;

- $\mathrm{SC}-\mathrm{CO}_{2}$ is capable of separating compounds that are less volatile, have a higher molecular weight and/or are more polar as pressure increases ${ }^{[2]}$.

Special applications of supercritical fluids to food processing: As mentioned before carbon dioxide is the most common supercritical fluid in the food industry. Due to the non-toxicity and low critical temperature, it can be used to extract thermally labile food components and the product is not contaminated with residual solvent. Further, the extract's color, composition, odor, texture are controllable and extraction by supercritical fluid carbon dioxide retains the aroma of the product. Supercritical fluid extraction provides a distinct advantage not only in the replacement but also extracts oils that are lower in iron and free fatty acid. Some application of SFE in food is mentioned below:

Removal of fat from foods: Edible oils and their components has been the target of supercritical fluid processing since the early 70s. Although triacylglycerides are only fairly soluble in $\mathrm{SC}-\mathrm{CO}_{2}$, the advantages of organic solvent-free processing have stimulated research and development in various areas. One of these is the removal of fat from food. The process has been fully designed for commercial application, using the aforementioned standard design. The process has the advantage of producing fat-free or fat-reduced potato chips. According to the expected taste the amount of remaining fat in the potato chips can easily be controlled ${ }^{[7,8]}$.
Enrichment of vitamin $\mathbf{E}$ from natural sources: SFE offers several advantages for the enrichment of tocochromanols over conventional techniques such as vacuum distillation, in particular a lower operating temperature. As starting material one can use various edible oils or their distillates. Most promising as feed materials are $\mathrm{CPO}$ ) and $\mathrm{SODD}^{[9]}$. CPO contains several tocotrienols and tocopherols at a total concentration of approximately $500 \mathrm{ppm}$. SODD may contain (after several conventional concentration steps) about 50\% tocopherols. Both materials can be used for the production of enriched fractions of tocochromanols ${ }^{[8,9]}$. Although it is possible to recover tocochromanols directly from CPO, it is better to produce esters of the triglycerides in order to be able to more easily separate these compounds from the tocochromanols. In this method, the triglycerides are subject to an esterification with methanol to form fatty acid methyl esters, which are easily extractable with $\mathrm{CO}_{2}$. That means that the tocochromanols, together with other unsaponifiable matter such as squalene and sterol are enriched in the bottom phase of an extraction column. This attempt is described in more detail by. For a discussion of enriching tocochromanols, phase equilibrium data have to be considered first ${ }^{[10]}$. FFA and tocochromanols exhibit a much higher solubility in $\mathrm{CO}_{2}$ than the triglycerides. Hence, these components are enriched in the gaseous phase, expressed by a distribution coefficient being higher than one. The distribution coefficient of the triglycerides is smaller than one, whereas that for the carotenes is much smaller than one, meaning that these components stay in the liquid oil phase. Thus, tocochromanols can be extracted as the top phase product in a separation column, whereas carotenes remain in the bottom phase product together with triglycerides. For recovering the carotenes together with the tococromanols the above mentioned esterification to volatile $\left(\mathrm{CO}_{2}\right.$ soluble) methyl esters makes possible to recover tocochromanols and carotenes (together with squalene and sterols) as bottom product from this natural source ${ }^{[9,11]}$. When the glycerides (in case of the esterification) or the FFAs from deodorizer distillates have been removed, then there is a feed material available for obtaining enriched fractions of tocochromanols and carotenes of much higher concentration. In this feed material, tocochromanols and carotenes (in case of palm oil) are the main components and have to be separated from other unsaponifiable substances present, such as squalene and sterols. Of these compounds, squalene has the highest solubility in $\mathrm{SC}-\mathrm{CO}_{2}$, all phytosterols have a 
rather low solubility in $\mathrm{CO}_{2}$ (and remain in the oil phase), and tocochromanols exhibit an intermediate solubility between the two. In a second separation step tocochromanols are separated from phytosterols. A further purification of these compounds is possible, e.g. with adsorptive orchromatographic techniques, again using supercritical fluids ${ }^{[2,10]}$.

Removal of alcohol from wine and beer, and related applications: De-alcoholized wine or beer is achieved by removing ethanol from water. Distillation is well known for this purpose with the disadvantage that aroma compounds will also be removed. New techniques like membrane separation (pervaporation) emerge, and in between these is SFE with $\mathrm{CO}_{2}{ }^{[1]}$.

Starting from an aqueous solution with about $10 \%$ $(\mathrm{w} / \mathrm{w})$ ethanol, ethanol can be removed by $\mathrm{SC}-\mathrm{CO}_{2}$ in a stripping column. The rate of ethanol removal depends strongly on temperature. Reducing the alcohol content to values well below $0.5 \%(\mathrm{w} / \mathrm{w})$ requires about $2.5 \mathrm{~h}$ at $45^{\circ} \mathrm{C}$ under non-optimized conditions. Much shorter times for the ethanol removal can be obtained if flow rates and mass transfer equipment are carefully selected. With the information available in the literature, for instance from, a column for dealcoholizing aqueous solutions can be designed. Recovery of aroma compounds is achieved by a side column in which a separation from ethanol is carried out $^{[2,1]}$.

A related process that can be mentioned is the recovery of absolute alcohol. Many studies were carried out at conditions of complete miscibility of ethanol and $\mathrm{CO} 2$ in order to get a high solubility of ethanol in the vapor phase. At these conditions, anhydrous ethanol cannot be produced. However, ethanol can be concentrated above azeotropic composition whenever the pressure in the ternary mixture $\mathrm{CO}_{2}+$ ethanol + water is below the critical pressure of the binary mixture $\mathrm{CO}_{2}+$ ethanol ${ }^{[2]}$.

Encapsulation of liquids for engineering solid products: A liquid product can be entrapped by adsorption onto solid particles (liquid at the outside of solid particles), by agglomeration (liquid in the free volumes between the solid particles), or by impregnation (liquid within the pore system of the solid particles). Microspheres or larger capsules can be formed, totally encapsulating the liquid. The solid material provides a coating for the liquid inside. Such particulate products can be achieved by means of supercritical fluid processing ${ }^{[11]}$. An example is the socalled concentrated powder form-process, wherein $\mathrm{CO}_{2}$ is mixed (dissolved) in the liquid feed by static mixing. The $\mathrm{CO}_{2}$-liquid feed mixture is then sprayed into a spray chamber at ambient conditions together with the substrate material. The $\mathrm{CO}_{2}$ is suddenly released from the liquid, and the liquid forms small droplets. During the spraying process, solid substrate and liquid droplets are intensively mixed and combined to a solid particulate product of the type described above. The product is finally removed from the chamber as a free flowing powder and separated from the outgoing gas stream by a cyclone. With this type of process, a wide variety of solid substrates can be applied to uptake liquids of different kind and up to about $90 \%{ }^{[12]}$. As advantages can be claimed the easier handling and storage, prevention of oxidation processes, and easier dosage. Solid products can also be formed under high pressure conditions. As an example for such a type of process, the encapsulation or adsorption of tocopherol acetate on silica gel. Here, about $50 \%$ of tocopherol acetate can be incorporated onto the silica gel without apparent change of morphology and flow properties of the powder. The powder with $50 \%$ loading is still free flowing. The amount which can be adsorbed at high pressures is comparable to that of normal pressure. Only at very high densities, the equilibrium loading decreases. In the experiments the autoclave was used to saturate the $\mathrm{SC}-\mathrm{CO}_{2}$ current with tocopherol acetate, and the density of the solvent was changed in the nozzle where the loaded $\mathrm{SC}-\mathrm{CO}_{2}$ phase was fed to the absorber. This adsorption at high pressures makes possible the direct product formation in the supercritical fluid, with the advantageous effect that the supercritical solvent can easily be recycled without substantial compression $^{[2,11,12]}$.

Extraction and characterization of functional compounds: Nowadays, the growing interest in the socalled functional foods has raised the demand of new functional ingredients that can be used by the food industry. These functional ingredients are preferred to have natural origin and to have been obtained using environmentally clean extraction techniques. As expected, the complexity of the natural ingredients with biological activity is very high; this fact has lead to the development of new methodologies to extract and characterize them. In order to preserve the activity of such ingredients and to prevent changes in the chemical composition of the functional compounds and/or mixture of compounds, sample preparation techniques based on the use of compressed fluids have been widely developed. SFE has been used to obtain extracts with 
antioxidant activity from microalgae; by using the combination of SFE and HPLC with both DAD ESI$\mathrm{MS}^{[13]}$. Several functional compounds were identified corresponding to different carotenoids along with chlorophyll a and some chlorophyll degradation products. These compounds could be associated to the biological activity of such extracts ${ }^{[14,11]}$. Supercritical $\mathrm{CO}_{2}$ has also been used to extract and characterize antimicrobial compounds and food preservatives from microalgae. Carotenoids are a group of compounds of great importance to human health since they can act, e.g., as potent antioxidants; however, due to their chemical characteristics they are easily degraded by temperature or oxygen, so, the use of SFE has been suggested to minimize risks of activity lost being thus applied to the extraction of carotenoids from different matrices $^{[15]}$. In this application, a vegetable oil was also used as co-solvent showing an improvement in the extraction yield as well as in the stability of the pigment. In both cases, the use of oils as co-extracting agents presents an important drawback that is the elimination of oil. It helps to improve the extraction but the extract is a mixture of the extracted components of the oil and the "pure" extract. In general terms, the use of SFE allows the analysis of essential oil preserving its integrity, without the formation of off flavors that could interfere in the characterization of the sample. As it was told before in order to widen the range of application of SFE to relatively polar compounds, small amounts of modifiers $(\leq 15 \%)$ are added to carbon dioxide allowing the extraction of more polar substances ${ }^{[11]}$.

Other examples of the extraction of valuable compounds from foods using SFE are the isolation of cholesterol from cattle brains and fat-soluble vitamins from parmigiano regiano cheese. The main problem with cattle brains, as well as many other raw food matrices is its high content in water. Water can interfere in the extraction process in two ways: lixiviation or acting as co-solvent thus interfering in the reproducibility of the extraction procedure. In order to avoid this situation, the most common strategy is drying or freeze drying the sample prior to extraction although some authors prefer to mix the sample with a water absorbent inside the extraction cell, for example magnesium sulphate ${ }^{[16,3]}$. Another problem related to the extraction of real food samples is their variable fat content which can also interfere in the extraction of the target compounds due to a coextraction of the fat at the selected extraction conditions. Two main approaches have been used to overcome this problem, the first one uses a fat retainer, mainly basic alumina, neutral alumina, florisil or silica, placed in a separate chamber downstream the extraction thimble, while the other one uses the fat retainer inside the extraction cell ${ }^{[3]}$.

Application of SFE in food safety: At present, food safety includes many different issues such as detection of frauds, adulterations and contaminations. Among these topics, detection of food pollutants is important not only for consumers but also for administrations, control laboratories, and regulatory agencies. In order to protect consumers' health, regulations establish strict limits to the presence of pollutants in foods that must be carefully observed and determined. Generally, the analysis of food pollutants is linked to long extraction and cleanup procedures commonly based on the use of, e.g., soxhlet and/or saponification. These procedures are laborious and time consuming and, besides, usually employ large volumes of toxic organic solvents. With the objective of reducing both, the sample preparation time and the massive use of organic solvents, techniques based on compressed fluids such as SFE have been developed. One of the main areas of application of SFE in the last few years has been in food pollutants analysis, mainly pesticide residues and environmental pollutants ${ }^{[5]}$.

Several methods has been developed for the analysis of multiple pesticides (organochlorine, organophosphorus, organonitrogen and pyrethroid) in potatoes, tomatoes, apples, lettuces and honey with a single cleanup step using supercritical $\mathrm{CO}_{2}$ modified with $10 \%$ of acetonitrile. Similar study have been carried out for the analysis of multiresidues of pesticides, using SFE as a cleanup step, in cereals, fish muscle, vegetable canned soups, vegetables or infant and diet foods ${ }^{[5,3]}$.

A common characteristic of these study is the extremely high selectivity of SFE in the isolation of the low polarity pesticides; this fact makes SFE probably the technique of choice to isolate pesticides from low fat food. As mentioned in the introduction, to correctly asses the concentration of an analyte in a food sample, a quantitative recovery should be obtained that will mostly depend on the recovery of the analytes and not on the extraction itself. To improve the recovery of the pollutants, a common strategy is the use of solid traps. These traps consist on a solid phase compatible with the analyte and are flushed away with a compatible solvent. The trapping step is very important in SFE method development not only because its effect in the quantitative recovery of the analytes but also because an extra selectivity can easily be introduced, especially in the case of solid-phase trapping, avoiding the use of further post-extraction clean up. Supercritical carbon 
dioxide extraction can advantageously be used to extract non-polar pollutants, such as PAH from foods. Different extraction and cleanup methods have been used, but the extracting conditions turned to be very similar (around $300 \mathrm{bar}$ and $100^{\circ} \mathrm{C}$ ) to optimize the PAH extraction ${ }^{[3]}$.

SFE and analytical uses: $\mathrm{SC}-\mathrm{CO}_{2}$ has been utilized in multiple methods of analysis. It is used as either an extraction medium, as in rapid analyses for fat content, or as a mobile phase, as in supercritical fluid chromatography. accordingly, the use of supercritical fluids in the detection of fat content, pesticide residues, and supercritical fluid chromatography as well as some analytical applications are highlighted below:

Rapid analysis for fat content: SFE have been used to determine the fat content of numerous products ranging from beef to oil seeds and vegetables. For the analysis of fats content in soybeans, sunflower, safflower, cottonseed, rapeseed and ground beef, it was found that supercritical fluid extraction yielded higher recoveries than those obtained by the AOCS approved methods. The use of an in-line piezoelectric detector is able to measure the change in weight of the sample during the extraction process. This allows for more accurate determination of the final weight of the sample after all of the fat has been extracted (total fat). In addition, it can allow for more rapid determination of the total fat by determining the point when the steady state mass has been reached without having to re-extract the sample multiple times to confirm that the steady state mass has been reached ${ }^{[5]}$.

Rapid analysis for pesticides in foods: Pesticide residues are a concern among consumers throughout the United States and other countries. Currently the methods of analyzing food products and other substances such as contaminated soil and water involve the use of organic solvents such as hexane and dichloromethane to extract the pesticides from the sample matrix. Once the pesticides have been extracted from the sample matrix, the samples must be "cleaned" to remove any unwanted compounds, such as lipids, which may interfere $\mathrm{GC}$ analysis of the sample for any pesticides present ${ }^{[17]}$. The most common method for cleaning is solid phase extraction. Supercritical fluid extraction provides an alternative to using organic solvents for the extraction of pesticides from their sample matrix. Some of the advantages which supercritical fluid extraction provides over the traditional methods of pesticide extraction are that the extraction can be performed in less time, and utilizes less solvent volume. In addition, supercritical fluid extraction can be tailored to the solute of interest by changing the temperature and pressure of the extraction process. Supercritical fluid extraction can also be tailored for pesticides that contain more polar groups by the addition of polar modifiers to the $\mathrm{CO}_{2}$ such as methanol ${ }^{[5,17]}$.

\section{New applications}

Supercritical particle formation: In the 1970s and 1980 s, supercritical processes were mainly focused on extraction and separation but, within the last 10 years, interest in supercritical fluid processing has also arisen in other areas. Here some of the new applications of SFE are mentioned

Nanoparticle formation using supercritical fluids: SFE were used in a number of different circumstances for the preparation of micro-nanodispersed organic systems. Of industrial use are their special solvent properties and the technical feasibility of large temperature gradients ${ }^{[18]}$. The generation of a sufficiently high super saturation for the initiation of a precipitation reaction with conventional solvents is on the one hand limited by the generally low dependency of the solubility on temperature, on the other by the difficulty to realize technically the necessary rapid heat exchange. With this in mind one process in which liquid $\mathrm{CO}_{2}$ is used as cooling agent deserves attention. In this so-called contact cooling process the active compound solution at $-78{ }^{\circ} \mathrm{C}$ is sprayed into a $\mathrm{CO}_{2}$ stream and particle formation is induced in the spray droplets by crystallization. From the viewpoint of toxicological acceptability, the non-combustibility, and the favorable critical data of $\mathrm{CO}_{2}\left(\mathrm{pc}=74 \mathrm{bar}, \mathrm{Tc}=31^{\circ} \mathrm{C}\right)$ the so-called RESS process (rapid expansion of supercritical solutions) appears particularly attractive, especially because extra process steps to remove residual solvent may no longer be required. However, SF- $\mathrm{CO}_{2}$ can act as an oxidizing agent with oxidationsensitive compounds such as $\beta$-carotene and is thus ruled out as a precipitation medium. Both in the GAS process (gas antisolvent process) and in the PCA process, $\mathrm{SF}-\mathrm{CO}_{2}$ instead of water serves as the precipitation medium from organic solvents. In the physically related SEDS the organic active-compound solution and the SF- $\mathrm{CO}_{2}$ are brought into contact in a coaxial mixing nozzle, and thus a rapid extraction of the solvent (e.g. acetone) is possible. However, to date there have only been reports of particle formation in the micrometer range ${ }^{[18,19]}$. 
Supercritical drying: Supercritical drying goes beyond the critical point of the working fluid in order to avoid the direct liquid-gas transition seen in ordinary drying. It is a process to remove liquid in a precisely controlled way, similar to freeze drying. It is is commonly used in the production of aerogel and in the preparation of biological specimens for scanning electron microscopy. As a substance crosses the boundary from liquid to gas (phase diagram), the substance volatilizes and so the volume of the liquid decreases. As this happens, surface tension at the solid-liquid interface pulls against any structures that the liquid is attached to. Delicate structures, like cell walls, the dendrites in silica gel and the tiny machinery of microelectromechanical devices, tend to be broken apart by this surface tension as the interface moves by. To avoid this, the sample can be brought from the liquid phase to the gas phase without crossing the liquid-gas boundary on the phase diagram; in freeze-drying, this means going around to the left (low temperature, low pressure). However, some structures are disrupted even by the solid-gas boundary. Supercritical drying, on the other hand, goes around the line to the right, on the high-temperature, high-pressure side. This route from liquid to gas does not cross any phase boundary, instead passing through the supercritical region, where the distinction between gas and liquid ceases to apply.

Fluids suitable for supercritical drying include carbon dioxide and freon. Nitrous oxide has similar physical behavior to carbon dioxide, but is a powerful oxidizer in its supercritical state. Supercritical water is also a powerful oxidizer, partly because its critical point occurs at such a high temperature $\left(374{ }^{\circ} \mathrm{C}\right)$ and pressure $\left(647^{\circ} \mathrm{K}\right.$ and $22.064 \mathrm{Mpa}^{[20]}$.

\section{CONCLUSION}

SFE has been suggested as the method of choice for thermo labile compounds extraction. It has been used for developing an ever-expanding niche in the food industry whether it is used as a solvent for extraction or analyses. Now a day, SFE is really needed as advance method which can provide fast, reliable, clean and cheap methods for routine analysis.

\section{REFERENCES}

1. Bravi, E., G. Pperretti, L. Motanari, F. Favati and P. Fantozzi, 2007. Supercritical fluid extraction for quality control in beer industry. J. Supercrit. Fluids, 42: 342-346. DOI:10.1016/j.supflu.2007.02.012

2. Brunner, G., 2005. Supercritical fluids: Technology and application to food processing. J. Food Eng., 67: 21-33. DOI:10.1016/j.jfoodeng.2004.05.060
3. José, A.M., H. Miguel, C. Alejandro and I. Elena, 2007. Use of compressed fluids for sample preparation. Food Appli. J. Chromatog. 1152: 234246. DOI:10.1016/j.chroma.2007.02.046

4. Dixon, D.J. and K.P. Johnston, 1997. Supercritical Fluids. In: Encyclopedia of Separation Technology, Ruthven, D.M., (Eds.). 1st Edn., John Wiley Interscience, New York, pp: 1544-1569. ISBN-10: 0471161241.

5. Rozzi, N.L. and R.K. Singh, 2002. Supercritical fluids and the food industry. J. Comprehensive Rev. Food Sci. Food Saf., 1:33-44. DOI: 10.1111/j.1541-4337.2002.tb00005.x About

6. Montañés, F., N. Corzo, A. Olano, G. Reglero, E. Ibáñez and T. Fornari, 2008. Selective fractionation of carbohydrate complex mixtures by supercritical extraction with $\mathrm{CO} 2$ and different co-solvents. J. Supercrit., 45: 189-194. DOI:10.1016/j.supflu.2007.08.012

7. Catchpole, O.J., S.J. Tallon, J.B. Grey, K. Fletcher and A.J. Fletcher, 2008. Extraction of lipids from a specialist dairy stream. J. Supercrit. Fluids, 45: 314-321. DOI:10.1016/j.supflu.2008.01.004

8. Chuang. M.H. and G. Brunner, 2006. Concentration of minor components in crude palm oil. J. Supercrit. Fluids, 37: 151-156. DOI:10.1016/j.supflu.2005.09.004

9. Fang, T., M. Goto, Wang, Xianbao, X. Ding, J. Geng, M. Sasaki and T. Hirose, 2007. Separation of natural tocopherols from soybean oil byproduct with supercritical carbon dioxide. J. Supercrit. Fluids, 40: 50-58. DOI:10.1016/j.supflu.2006.04.008

10. Zeng, A.W., X.G. Yuan and K.T. Yu, 2008. Extraction of soybean isoflavones from soybean meal with aqueous methanol modified supercritical carbon dioxide. J. Food Eng., 89: 384-389. DOI:10.1016/j.jfoodeng.2008.05.004

11. Pessoa, F.L.P. and A.M.C. Uller, 2002. An economic evaluation based on an experimental study of the vitamin $\mathrm{E}$ concentration present in deodorizer distillate of soybean oil using supercritical $\mathrm{CO}_{2}$. J. Supercrit. Fluids, 23: 257265. DOI:10.1016/S0896-8446(01)00140-1

12 Patrick, J.G., J.W. Martin, M.S. Kevin and M.H. Steven, 2005. Drug delivery goes supercritical. J. Materials Today, 8: 42-48. DOI:10.1016/S1369-7021(05)71036-1

13. Wang,Y., W. Yiping, J. Yang, R. Pfeffer, R. Dave and B. Michniak, Y. Wang, et al., 2006. The application of a supercritical antisolvent process for sustained drug delivery. J. Powder Technol., 164: 94-102. DOI:10.1016/j.powtec.2006.03.004 
14. Franceschi, E., A.M. De Cesaro, M. Feiten, S.R.S. Ferreira, C. Dariva, M.H. Kunita, A.F. Rubira, E.C. Muniz, M.L. Corazza and J.V. Oliveira, 2008. Precipitation of $\beta$-carotene and PHBV and co-precipitation from SEDS technique using supercritical $\mathrm{CO}_{2}$. J. Supercritical Fluids, 47: 256-259. DOI:10.1016/j.supflu.2008.08.002

15. Juan, C., O. Bernardo, Q. Nathalie and M. José, 2006. Solubility of carotenoid pigments (Lycopene and Astaxanthin) in supercritical carbon dioxide fluid. J. Phase Equilibria, 247: 90-95. http://cat.inist.fr/?aModele $=$ afficheN\&cpsidt $=1807$ 4549

16. Mantell, C., M. Rodriguez, A. Torres, F.A. Macias and E.J. Martinez, 2008. Supercritical fluid extraction of bioactive compounds from sunflower leaves with carbon dioxide and water on a pilot plant scale. J. Supercrit. Fluids, 45: 37-42. DOI:10.1016/j.supflu.2007.12.002
17. Cortes, J.M., 2009. Pesticide Residue Analysis by RPLC-GC in Lycopene and other Carotenoids Obtained from Tomatoes by Supercritical Fluid Extraction. J. Food Chemistry 113: 280-284. DOI:10.1016/j.foodchem.2008.07.010

18. Riegr, J. and D. Horn, 2001. Organic nanoparticles in the aqueous phase-theory, experiment and use. J. Angew. Chem. Int. Ed., 40: 4330-4361. DOI.wiley.com/10.1002/15213773(20011203)40:23\%3C4330::AIDANIE4330\%3E3.0.CO;2-W -

19. Herrero, M., A. Cifuentes, E. Ibanez and M. Herrero, et al., 2006. Sub- and supercritical extraction of functional ingredients from different natural sources: Plants, food-by-products, algae and microalgae. J. Food Chem., 98: 136-148. DOI:10.1016/j.foodchem.2005.05.058. 